\title{
Radiation-Pressure Cooling of Bound Resonant Absorbers
}

\author{
D. J. Wineland, R. E. Drullinger, and F. L. Walls \\ Time and Frequency Division, National Bureau of Standards, Boulder, Colorado 80303
} (Received 26 April 1978)

\begin{abstract}
We report the first observation of radiation-pressure cooling on a system of resonant absorbers which are elastically bound to a laboratory fixed apparatus. $\mathrm{Mg}$ II ions confined in a Penning electromagnetic trap are cooled to $<40 \mathrm{~K}$ by irradiating them with the $8-\mu \mathrm{W}$ output of a frequency doubled, single-made dye laser tuned to the low-frequency side of the Doppler profile on the ${ }^{2} S_{1 / 2} \leftrightarrow{ }^{2} P_{3 / 2}\left(M_{J}=+\frac{1}{2} \leftrightarrow M_{J}=+\frac{3}{2}\right.$ or $\left.M_{J}=-\frac{1}{2} \leftrightarrow M_{J}=-\frac{3}{2}\right)$ transitions. Cooling to approximately $10^{-3} \mathrm{~K}$ should be possible.
\end{abstract}

Any scheme to improve fundamentally the resolution and accuracy of high-resolution spectroscopy (rf as well as optical) beyond the present limits must incorporate sub-Doppler techniques and a means to suppress substantially the secondorder and residual first-order Doppler effects. In the following we report the first experimental evidence that bound absorbers can be cooled via radiation pressure thereby fundamentally reducing both the first- and second-order Doppler effects. The use of radiation pressure for cooling was independently suggested for the case of a gas of neutral atoms $\mathrm{s}^{1}$ and for ions bound in an electromagnetic trap ${ }^{2}$ and has also been incorporated into the suggested schemes for optical trapping. ${ }^{3,4}$ Methods for significantly cooling electrons and ions contained in electromagnetic traps via radiation damping or collisions with cold gas have been known for some time $\mathrm{s}^{5-7}$; however, these techniques do not provide cooling of atomic ions much below room temperature. It has recently been demonstrated ${ }^{8}$ that the magnetron motion of electrons in a Penning trap can be cooled by nonlinear excitation of the other degrees of freedom. The method of radiation-pressure cooling demonstrated here offers the possiblity to cool substantially all degrees of freedom.

The method is outlined for the case of a harmonically bound resonant absorber (resonance frequency $\nu_{0}$ ) which is constrained to move along the $x$ axis. We assume that its velocity is given by $v_{x}=v_{0} \cos 2 \pi \nu_{v} t$ where $\nu_{v}$ is its vibrational frequency and that the natural linewidth $(\Delta \nu)$ is less than $\nu_{v}$. The observed spectrum of the absorber in the laboratory contains the central resonance line with first-order-Doppler-effect-generated sidebands separated by $\nu_{v}$ having intensity $J_{n}^{2}\left(v_{0} \nu_{0} /\right.$ $c \nu_{v}$ ) (with $n$ a positive or negative integer) when observed in the direction of motion; here, $J_{n}$ is the Bessel function of order $n$. If we irradiate the absorber with photons of frequency $\nu_{L}=\nu_{0}$ $+n \nu_{v}$, the frequencies of the resonantly scattered photons occur at $\nu_{0}$ and symmetrically around $\nu_{0}$ at the sideband frequencies $\nu_{0} \pm \nu_{v}, \nu_{0} \pm 2 \nu_{v}, \ldots$. Therefore, although photons of energy $h\left(\nu_{0}+n \nu_{v}\right)$ are absorbed, on the average photons of energy $h \nu_{0}$ are reemitted; when $n$ is negative, this energy difference causes the kinetic energy of the absorber to decrease by $h h \nu_{v} \mid$ per scattering event. In our experiments, $\Delta \nu \gg \nu_{v}$; however, the above conclusion is still valid. An alternative explanation is that when $\nu_{L}<\nu_{0}$, the absorber predominantly interacts with the incident radiation when it moves towards the source of radiation and Doppler shifts the frequency into resonance, i.e., when $\nu_{L}\left(1+v_{x} / c\right)=\nu_{0}$. In the absorption process, the photon momentum is first transferred to the absorber causing its momentum to change by $h / \lambda$ $\left(\lambda \nu_{L}=c\right)$ and since the emission occurs symmetrically in the $\pm x$ directions, the net effect is to change the velocity of the absorber by $\Delta v_{x}=h / M \lambda$ ( $M=$ mass of absorber). If $\Delta v_{x} \ll v_{x}$, then the kinetic energy of the absorber decreases by an amount $M v_{x} \Delta v_{x}=n h \nu_{v}$. Through the harmonic binding force, virtually all of the photon recoil momentum is eventually transferred to the "lattice" (trap structure in this case); however, since the mass of this lattice is very large, the energy change occurs in the vibrational motion. Note that, when $\Delta \nu<\nu_{v}$, scattering of photons with energy $h \nu_{0}$ is equivalent to the Mössbauer effect.

In our experiments we store approximately 5 $\times 10^{4} \mathrm{Mg}$ II ions (density approximately $2 \times 10^{7} /$ $\mathrm{cm}^{3}$ ) in a copper Penning tap with hyperbolic electrodes of characteristic dimensions ${ }^{5.6} \quad r_{0}=1.64 z_{0}$ $=0.63 \mathrm{~cm}$. Typical operating parameter $\mathrm{s}$ are $v_{0}$ $=7 \mathrm{~V}$ and $B_{0}=1.3 \mathrm{~T}$. The motion of an ion in the trap is comprised of a harmonic oscillation along the axial $(z)$ direction (frequency $\nu_{z}$ ) plus a composite of circular cyclotron motion (frequency $\nu_{c}{ }^{\prime}$ ) superimposed upon a circular magnetron motion (frequency $\nu_{m}$ ) in the $x-y$ plane. In the notation of 
Byrne and Farago,

$$
\begin{aligned}
\overrightarrow{\mathrm{r}}=x+i y & =\overrightarrow{\mathrm{r}}_{c}+\overrightarrow{\mathrm{r}}_{m} \\
& =\overrightarrow{\mathrm{r}}_{c_{0}} \exp \left(i \omega_{c}^{\prime} t\right)+\overrightarrow{\mathrm{r}}_{m_{0}} \exp \left(i \omega_{m} t\right),
\end{aligned}
$$

where $\omega=2 \pi \nu$. For the above operating parameters, $\nu_{z} \simeq 205 \mathrm{kHz}, \nu_{c}^{\prime} \simeq 798 \mathrm{kHz}$, and $\nu_{m} \simeq 26$ $\mathrm{kHz}$.

The trap apparatus is mounted in an enclosure at high vacuum allowing observed ion thermalization times due to collisions with the background gas as long as $30 \mathrm{~min}$ and storage times of approximately 1 day. $\mathrm{Mg}$ is emitted from an oven and ions are formed by an electron beam coincident with the trap axis. Because the oven is approximately $0.5 \mathrm{~cm}$ from the trap, the temperature of the trap and therefore of the background gas is elevated above room temperature. Thermal motion of the ions induces currents in the trap electrodes yielding a signal proportional to $N T$ ( $N=$ ion number, $T=$ ion temperature).$^{5,6}$ Since the time for the cooling experiments is much less than the ion storage time and since observations confirm that the laser does not eject ions from the trap, $N$ can be assumed constant. Therefore the signal provides a direct measurement of ion temperature.

The radiation for the experiments is derived from the frequency-doubled output of a singlemode, c.w., Rhodamine-110 dye laser. In the spectral region of interest $(\sim 560 \mathrm{~nm})$, this laser has a power output of approximately $250 \mathrm{~mW}$ in a 1-MHz bandwidth when pumped with $3 \mathrm{~W}$ of $514.5-$ $\mathrm{nm}$ radiation from an $\mathrm{Ar}^{+}$laser. The dye laser can be continuously tuned across $30 \mathrm{GHz}$ and is frequency doubled into the uv with a $90^{\circ}$-phasematched $A D * P$ (deuterated ammonia dihydrogen phosphate) crystal. This allows us to tune across the ${ }^{2} S_{1 / 2} \rightarrow{ }^{2} P_{3 / 2}\left(M_{J}= \pm \frac{1}{2}-M_{J}= \pm \frac{3}{2}\right)$ transitions in one sweep. The uv output (up to $40 \mu \mathrm{W}$ ) is confined in a 1-2-mm-diam beam and is polarized perpendicular to the trap/magnet axis. It is introduced to and exits from holes in the ring electrode of the trap.

In a magnetic field the ${ }^{2} S_{1 / 2} \rightarrow{ }^{2} P_{3 / 2}$ transition splits according to the electronic Zeeman effect. The laser radiation is nearly uncoupled from the $\Delta M=0$ transitions due to the polarization; moreover, all of the $\left|M_{J}\right|=\frac{1}{2}-\left|M_{J}\right|=\frac{1}{2}$ transitions optically pump the ground state. However, the $M_{J}= \pm \frac{1}{2} \rightarrow M_{J}= \pm \frac{3}{2}$ transitions can be driven many times in succession and provide the transitions for cooling. Figure 1 (a) shows an experimental trace of ion temperature versus uv frequency
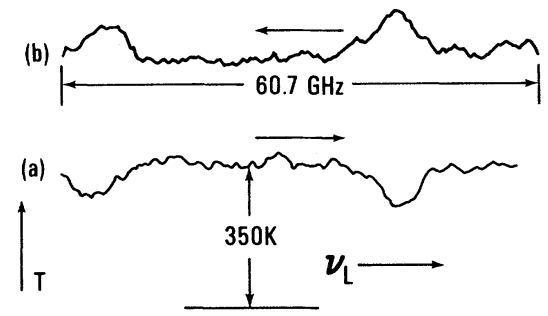

FIG. 1. Ion temperature vs swept laser frequency. Arrows above the traces show the direction of the sweep. The two resonance curves correspond to the ${ }^{2} S_{1 / 2} \leftrightarrow{ }^{2} P_{3 / 2}\left(M_{J}=-\frac{1}{2} \leftrightarrow M_{J}=-\frac{3}{2}\right.$ and $M_{J}=+\frac{1}{2} \leftrightarrow M_{J}$ $=+\frac{3}{2}$ ) transitions. When the laser frequency sweep is positive, the transitions are indicated by a temperature decrease; when the sweep is negative the transitions are indicated by an increase in temperature. Curve $b$ is displaced upward and the baseline is not shown.

when the laser is swept from low to high frequencies through the ${ }^{2} S_{1 / 2} \varpi^{2} P_{3 / 2}$ manifold. A decrease in temperature is observed at two frequencies corresponding to the separation of the $+\frac{1}{2}-+\frac{3}{2}$ and $-\frac{1}{2} \leftrightarrow-\frac{3}{2}$ transitions. As the radiation frequency is swept up through the low-frequency side of the Doppler profile on either transition, it cools the ions. When line center is reached, cooling stops; and as the laser sweeps through the high-frequency side of the Doppler profile, heating occurs. Conversely, if the laser frequency is swept down through the transitions, heating appears first and then cooling. [See Fig. 1(b).]

The rate of heat extraction (addition) per ion is given by

$$
d Q / d t=C h\left(\nu_{L}-\nu_{0}\right) \sigma_{s}\left(\nu_{L}\right) I\left(\nu_{L}\right) / h \nu_{L},
$$

where $I\left(\nu_{L}\right)$ is the laser flux $\left(\mathrm{W} / \mathrm{cm}^{2}\right)$ and $C$ is a factor $\leqslant 1$ describing the overlap of the laser beam with the ion cloud. The cross section $\sigma_{s}\left(\nu_{L}\right)$ is given by the convolution of the natural line shape with the Doppler profile. When $\Delta \nu$ is much less than the Doppler width, $\sigma_{s}\left(\nu_{\nu}\right)$ can be given by

$$
\sigma_{s}\left(\nu_{L}\right) \simeq \sigma_{0} \frac{\Delta \nu \sqrt{\pi}}{2 \nu_{\mathrm{D}}} \exp \left[-\left(\frac{\nu_{L}-\nu_{0}}{\nu_{\mathrm{D}}}\right)^{2}\right],
$$

where $\sigma_{0}$ is the resonance scattering cross section $\left(\frac{3}{2} \pi \lambda^{2}\right.$ for the transitions here) and where $\nu_{D}$ $\equiv(2 k T / M)^{1 / 2} \nu_{0} / c$. The monitored signal is proportional to the temperature of the strongly coupled cyclotron and axial motions but not to the magnetron motion. ${ }^{5,6}$ Moreover the laser only weakly couples to the magnetron motion (see below) and therefore each ion has a specific heat of $2 k$ ( $k=$ Boltzman's constant). Using Eqs. (2) 
and (3) the rate of temperature change is therefore

$$
\frac{d T}{d t}=\frac{1}{2 k} \frac{d Q}{d t}=C \frac{I\left(\nu_{L}\right) \sigma_{0} \Delta \nu \sqrt{\pi}\left(\nu_{L}-\nu_{0}\right)}{4 \nu_{\mathrm{D}} \nu_{L}{ }^{k}} \exp \left[-\left(\frac{\nu_{L}-\nu_{0}}{\nu_{\mathrm{D}}}\right)^{2}\right] \text {. }
$$

When the laser frequency is swept across the line so that $\nu_{L}-\nu_{0}=\dot{\nu} t\left(\boldsymbol{\nu} \equiv d \nu_{L} / d t\right)$ and when $I\left(\nu_{L}\right)$ equals a constant value $I_{0}$, then in the limit of small cooling (heating) where $\nu_{\mathrm{D}}$ is nearly constant, Eq. (4) can be integrated from $-\infty$ to $t$ to give

$$
\boldsymbol{T}(t)=T\left(\nu_{L}-\nu_{0}\right) \simeq \mp C I_{0} \sigma_{0} \frac{\sqrt{\pi} \Delta \nu \nu_{\mathrm{D}}}{8 \dot{\nu} \nu_{0} k} \exp \left[-\left(\frac{\nu_{L}-\nu_{0}}{\nu_{\mathrm{D}}}\right)^{2}\right],
$$

where the sign is negative for sweeping up and positive for sweeping down. The maximum cooling rate is expected when $\nu_{L}=\nu_{0}-\nu_{D} \sqrt{2}$. Note that for small temperature changes the observed line shape (temperature ver sus frequency) should reproduce the normal Doppler line shape. For the curves in Fig. 1, $I_{0} \simeq 4 \mu \mathrm{W} / \mathrm{mm}^{2}$ for the $-\frac{1}{2} \rightarrow-\frac{3}{2}$ and $6 \mu \mathrm{W} / \mathrm{mm}^{2}$ for the $+\frac{1}{2} \rightarrow+\frac{3}{2}$ transitions, $C$ $\simeq 0.5, \nu^{\prime}=0.1 \mathrm{GHz} / \mathrm{sec}$, and $^{10} \Delta \nu \simeq 43 \mathrm{MHz}$, yielding a factor $250 \mathrm{~K}$ in front of the exponential in Eq. (5). The ambient (trap) temperature was measured to be approximately $350 \pm 50 \mathrm{~K}$ by infrared means. The observed cooling (heating) $(\sim 100 \mathrm{~K})$ is not as strong as predicted which may be caused by the observed presence of other ions in trap which reduces the net effect or by too high an estimate of $C$. The observed splitting of the lines $(\simeq 37 \mathrm{GHz})$ agrees with the value predicted based on magnetic field measurements to about $3 \%$. The mean frequency of the two lines agrees with the reported value ${ }^{11}$ to within $0.02 \mathrm{~cm}^{-1}$ by comparison of the dye-laser output to the spectrum of $I_{2}$. The observed width of the lines $(4.5 \mathrm{GHz})$ is larger than that predicted from the ambient temperature $(2.9 \mathrm{GHz})$ and is partially caused by the magnetron-motion Doppler broadening and possibly the slightly shifted resonances of the ${ }^{25} \mathrm{Mg}$ and ${ }^{26} \mathrm{Mg}$ isotopes (about $10 \%$ each). We note, however, that if we cool the ions to one-half of their equilibrium temperature, and then sweep through the transition, the linewidth decreases by a factor of approximately 1.5 as expected.

In Fig. 2 a plot of temperature versus time is shown for the case when $\nu_{L}-\nu_{0}$ is constant and the laser is turned on for a fixed time as shown. The ions had previously been heated with the laser to about $700 \mathrm{~K}$. Laser cooling is then implemented for a fixed time showing the ion temperature approaching $0 \mathrm{~K}$ with an upper bound of $40 \mathrm{~K}$ determined by the noise. After the laser is turned off, the ions rethermalize as shown. With $I_{0}=8 \mu \mathrm{W}, \nu_{L}-\nu_{0} \simeq-(2 \pm 1) \mathrm{GHz}$, we measure a cooling rate of $5 \mathrm{~K} / \mathrm{sec}$ but predict a rate of $15 \pm \frac{5}{10} \mathrm{~K} / \mathrm{sec}$ based on Eq. (4) evaluated at $T=350$ K. For this trace the background gas pressure was purposely made higher to reduce the thermalization time.

The laser can couple to the magnetron motion and we must estimate this effect. If we use Eq. (1) and its time derivative we can relate a given velocity change, $\vec{\Delta} v$, to a change in magnetron radius by the expression $\vec{\Delta} r_{m}=i \Delta \vec{v} /\left(\omega_{c}^{\prime}-\omega_{m}\right)$. For each scattering event $\Delta \overrightarrow{\mathrm{v}}=\hat{x} h / M \lambda$ which causes the magnetron orbit size to change by $\left|\Delta \overrightarrow{\mathrm{r}}_{m}\right| \simeq 1$ $\times 10^{-6} \mathrm{~cm}$. If we irradiate the ion cloud uniformly, $\left|\overrightarrow{\mathrm{r}}_{m}\right|$ increases in a radom walk with step size $\left|\Delta \overrightarrow{\mathbf{r}}_{m}\right|$; this is a small effect. Moreover, if one irradiates the cloud preferentially on the side of the trap where the magnetron motion recedes from the laser, the magnetron vector should become smaller. This can be used to overcome the diffusion of ions out of the trap due to collisions and therefore indefinite confinement should be possible. We have mapped the ion cloud shape by measuring the cooling (heating) signal strengths a as a function of laser beam position and found $r_{m}(\max ) \simeq 1 \mathrm{~mm}$ and $z(\max ) \simeq 0.5 \mathrm{~mm}$.

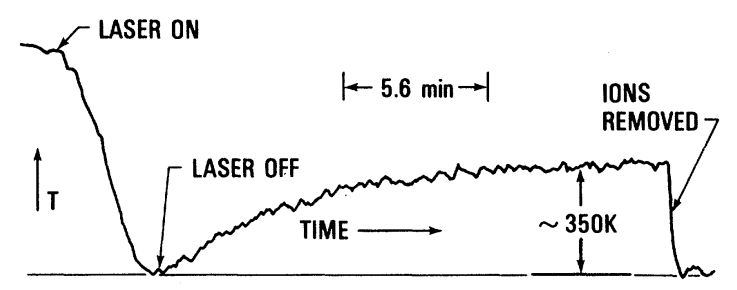

FIG. 2. Ion temperature vs time when laser cooling is applied for fixed $\nu_{L}-\nu_{0}$. The ions were initially heated above equilibrium temperature with the laser. Laser cooling was then applied on the $-\frac{1}{2} \longrightarrow-\frac{3}{2}$ transition for a fixed time until a temperature approaching $0 \mathrm{~K}(<40 \mathrm{~K})$ was achieved. After the laser is turned off, the ions rethermalize to the ambient temperature. 
The ultimate limit on cooling is determined by a competition of the damping rate on the vibrational energy due to laser cooling and the "noise" excitation of the vibrational energy due to the random occurrence (in time) of the photon impulses. In the limit where $\nu_{v} \ll \Delta \nu$ and when one tunes for maximum cooling $\left(\nu_{L}-\nu_{0}=-\frac{1}{2} \Delta \nu\right)$, the resulting kinetic energy is approximately equal to $h \Delta \nu / 8$ corresponding to $T \simeq 0.5 \times 10^{-3} \mathrm{~K}$ for the transitions discussed here. One can use the scattered photons from the cooling process as a monitor in a double-resonance experiment. Since the laser is not needed for trapping, it can be turned off for a relatively long time while the resonance of interest is probed. The possiblity also exists to use a mixture of ions-one kind which can be laser cooled and by collisions cools the other kind which are the ions of spectroscopic interest. We note that the cooling method is quite general and can in principle be applied to other cases such as ions or nuclei bound in solid lattice.

The authors wish to acknowledge the help of the other members of the Time and Frequency Division, in particular the support and leadership of H. Hellwig, and electronics construction by C. Klepper. The basic trap structure was built by one of the authors (D.J.W.) and R. S. VanDyck, Jr., at the University of Washington and was used with the kind permission of H. G. Dehmelt. The cooling limit was estimated with key input from E. M. Purcell and D. J. Larson, Harvard University. This work was funded in part by the U. S. Office of Naval Research under Contract No. N00014-77-F-0046.

\footnotetext{
${ }^{1}$ T. W. Hänsch and A. L. Schawlow, Opt. Commun。 13, 68 (1975).

${ }^{2}$ D. J. Wineland and H. Dehmelt, Bull. Am. Phys. Soc. 20,637 (1975).

${ }^{3}$ A. Ashkin, Phys. Rev. Lett. 24, 156 (1970), and 25, 1321 (1970), and $\underline{40}, 729$ (1978).

${ }^{4}$ V. S. Letokhov, Comments At. Mol. Phys. 6, 119

(1977), and many earlier references contained herein.

${ }^{5}$ H. G. Dehmelt and F. L. Walls, Phys. Rev. Lett. 21, 127 (1968); H. Dehmelt, in Advances in Atomic and Molecular Physics, edited by D. R. Bates and I. Esterman (Academic, New York, 1967, 1969), Vols. 3 and 5; D. J. Wineland and H. G. Dehmelt, J. Appl. Phys. $\underline{46}$, 919 (1975).

${ }^{6}$ R. A. Heppner, F. L. Walls, W. T. Armstrong, and G. H. Dunn, Phys. Rev. A 13, 1000 (1976).

${ }^{7}$ D. A. Church and H. G. Dehmelt, J. Appl. Phys. $\underline{40}$, 3421 (1969).

${ }^{8}$ R. S. VanDyck, Jr., P. B. Schwinberg, and H. G. Dehmelt, in Proceedings of Orbis Scientiae, Coral Gables, 1978 (Plenum, New York, to be published).

${ }^{9}$ J. Byrne and P. S. Farago, Proc. Phys. Soc. (London) 86,801 (1965).

${ }^{10}$ W. L. Wiese, M. W. Smith, and B. M. Miles, Atomic Transition Probabilities, U. S. National Bureau of Standards, National Standards Reference Data Series -22 (U. S. GPO, Washington, D. C., 1969), Vol. II.

${ }^{11} \mathrm{~S}$. Bashkin and J. O. Stoner, Jr., Atomic Energy Levels and Grotrian Diagrams (North-Holland, Amsterdam, 1975).
}

\title{
Collisional Electron Detachment of $\mathrm{H}^{-}$: A Complete Angular Distribution
}

\author{
M. G. Menendez and M. M. Duncan \\ Department of Physics and Astronomy, University of Georgia, Athens, Georgia 30602
} (Received 17 April 1978)

The doubly differential cross section resulting from electron loss of $0.5-\mathrm{MeV} \mathrm{H}^{-}$ions in collisions with $\mathrm{Ar}$ were measured from $0^{\circ}$ to $173^{\circ}$. The singly differential cross section (SDCS), angular distribution, for the $v_{e} \approx v_{i}$ group was integrated over solid angle and normalized to the known total electron-loss cross section. This SDCS was found to be dissimilar to that predicted by an electron scattering model previously used to describe projectile ionization. However, the SDCS was found to resemble, with some notable differences, the elastic scattering of electrons from Ar.

Experimental information on spectral shapes and angular dependences are necessary for an understanding of the details of electron detachment processes. In a recent paper ${ }^{1}$ secondary-electron energy spectra, the doubly differential cross section (DDCS), resulting from electron loss of fast $\mathrm{H}^{-}$ions were measured in the angular range $0^{\circ}-15^{\circ}$. The ion energy was $0.5 \mathrm{MeV}$ and the target was Ar. As expected, in the laboratory frame, the DDCS for projectile ionization exhibit- 